\title{
Safeguards and pitfalls for Bioprosthetic or Native Aortic Scallop Intentional Laceration to Prevent latrogenic Coronary Artery Obstruction during transcatheter aortic valve replacement-the BASILICA technique
}

\author{
Christopher G. Bruce ${ }^{1}$, Adam B. Greenbaum ${ }^{2}$, Vasilis C. Babaliaros ${ }^{2}$, Toby Rogers ${ }^{1,3}$, Robert J. Lederman ${ }^{1}$, \\ Jaffar M. Khan ${ }^{1}$ \\ ${ }^{1}$ Cardiovascular Branch, Division of Intramural Research, National Heart, Lung, and Blood Institute, National Institutes of Health, Bethesda, MD, \\ USA; ${ }^{2}$ Division of Cardiology, Emory Structural Heart and Valve Center, Emory University Hospital Midtown, Atlanta, GA, USA; ${ }^{3}$ Section of \\ Interventional Cardiology, MedStar Washington Hospital Center, Washington, DC, USA \\ Correspondence to: Jaffar M. Khan, BM, BCh, PhD. Cardiovascular Branch, Division of Intramural Research, National Heart, Lung and Blood \\ Institute, National Institutes of Health, Bethesda, MD, USA. Email: Jaffar.khan@nih.gov.
}

\begin{abstract}
Coronary artery obstruction is a devastating complication of transcatheter aortic valve replacement (TAVR). Bioprosthetic or native Aortic Scallop Intentional Laceration to prevent Iatrogenic Coronary Artery Obstruction (BASILICA) is a transcatheter, electrosurgical technique that was developed to prevent obstruction due to sinus effacement or sinus sequestration. BASILICA creates a midline laceration of one (solo) or both (doppio) offending aortic leaflets and has been performed in over 1,000 patients at high risk for obstruction. The procedure has been studied in the prospective BASILICA IDE Trial and data from the International BASILICA Registry of 214 patients supports efficacy and safety; procedural success was achieved in $94.4 \%$ and at thirty days $95.3 \%$ were free from culprit coronary artery obstruction, all-cause mortality was $2.8 \%$ and disabling stroke was reported in only $0.5 \%$. In this review we discuss screening for patients at high risk for coronary artery obstruction, technical details related to performing the BASILICA procedure and how to troubleshoot a BASILICA procedure.
\end{abstract}

Keywords: Transcatheter aortic valve replacement (TAVR); coronary artery obstruction; BASILICA; transcatheter electrosurgery

Submitted Jul 05, 2021. Accepted for publication Sep 06, 2021.

doi: 10.21037/acs-2021-tviv-26

View this article at: https://dx.doi.org/10.21037/acs-2021-tviv-26

\section{Introduction}

Coronary artery obstruction in the setting of TAVR in native aortic valves is a rare $(0.7 \%$ incidence $)$ but devastating complication with a $41 \%$ thirty-day mortality (1). TAVR for degenerative bioprosthetic surgical valves (Valve-invalve) carries higher risk still with an incidence of at least $2.3 \%$, and for TAV-in-TAV potentially as high as $20 \%(2-5)$. We developed the Bioprosthetic or native Aortic Scallop
Intentional Laceration to prevent Iatrogenic Coronary Artery obstruction (BASILICA) procedure to prevent coronary artery obstruction in patients deemed to be high risk of coronary obstruction (6). In the following review we summarize predictors of and screening for patients at high risk of coronary artery obstruction, BASILICA procedural steps for single and double leaflet laceration, recent adjunctive techniques, troubleshooting and future directions. 


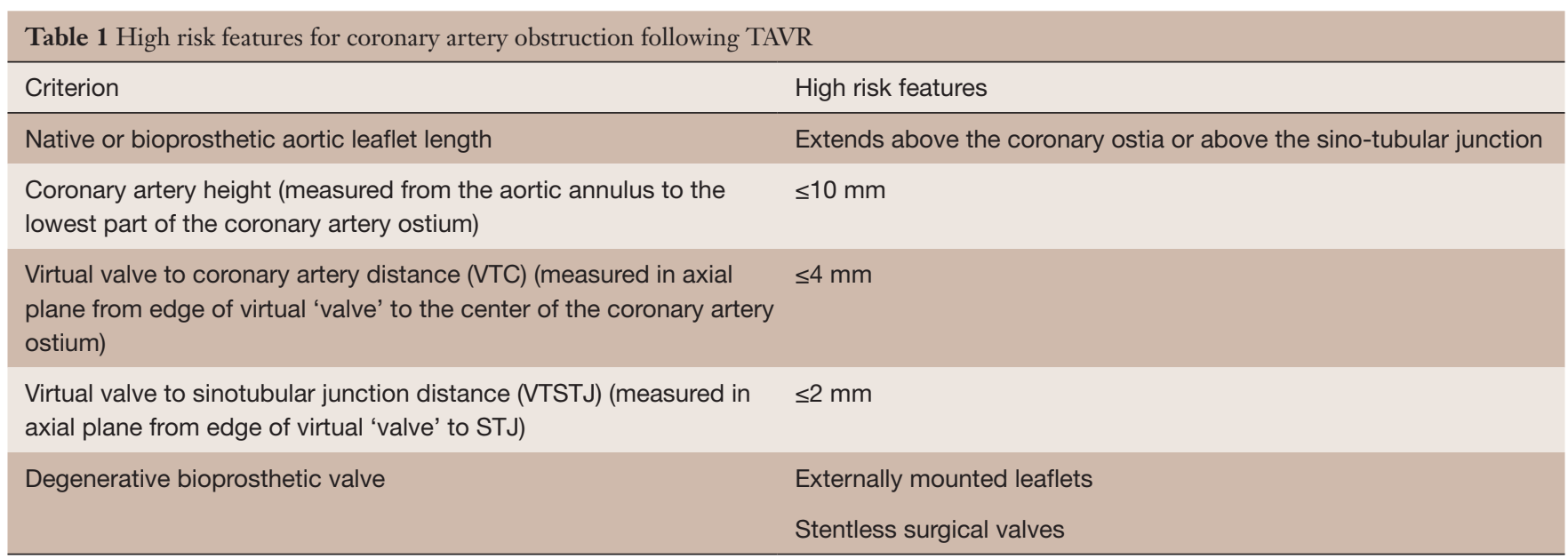

\section{Operative techniques}

\section{Preparation}

\section{Case selection and procedure planning}

BASILICA prevents coronary obstruction in patients at high risk for direct (ostial obstruction) or indirect (sinus sequestration) obstruction. Criteria for selecting patients at high risk for obstruction have been proposed and are including in Table 1.

Computed tomography (CT) should be performed in all cases to identify high risk anatomic features and obtain orthogonal fluoroscopic projections that can be used during the BASILICA procedure $(3,7)$. Importantly, if it is not possible to position the C-arm to achieve either CT predicted angle, craniocaudal angulation should be reduced whilst maintaining coronary artery alignment to the side or center for accurate catheter positioning and subsequent leaflet traversal.

\section{BASILICA procedure}

\section{Operation}

\section{Single ("solo") Leaflet BASILICA}

Solo BASILICA procedures are performed most commonly (8). Procedural steps are outlined in Figures 1 and 2. Right leaflet BASILICA is more technically challenging than left because optimal fluoroscopic angles are frequently difficult to achieve and catheter positioning is tricky.

\section{Double ("Doppio") Leaflet BASILICA}

Doppio leaflet laceration (Figure 3) combines laceration of left and right aortic leaflets. Technical complexity is increased with a greater number of procedural steps, multiple interacting and overlapping catheters, longer procedure times and increased contrast volumes. Following laceration of the second leaflet, THV deployment needs to be more expeditious than for solo procedures. For these reasons it is recommended that operators only undertake a doppio when they have developed competence with solo BASILICA, generally after two to three procedures. In the multi-center International BASILICA Registry, procedural success and adverse events including stroke, death and VARC-2 safety outcomes were not significantly different between doppio and solo procedures in experienced hands (8).

\section{Balloon-Augmented (BA) BASILICA}

Balloon augmentation (Figure 4) is a recent adjunctive technique that further enlarges leaflet splay area when performed in vitro. Following balloon-augmented laceration a rounded expansion towards the leaflet base enables a wider splay (9). BA-BASILICA has been reported in patients predicted to be at extreme risk of coronary artery obstruction despite conventional BASILICA, including TAV-in-TAV cases. BA-BASILICA was technically successful in all patients. When coronary artery stenting was required it was possible in a conventional, orthotopic fashion.

\section{Completion}

\section{Troubleshooting}

Meticulous adherence to the described procedural steps permits uncomplicated BASILICA in the majority of cases. Procedural cadence can be interrupted in several ways, outlined below, which have a common theme-failure to 
A

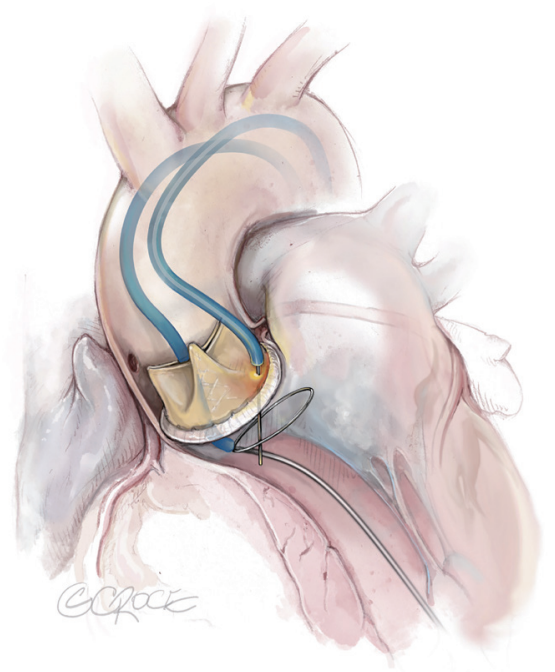

C

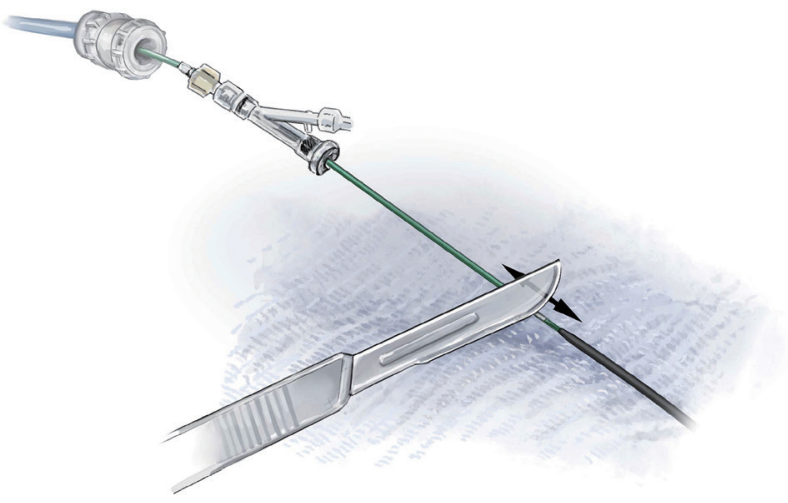

B
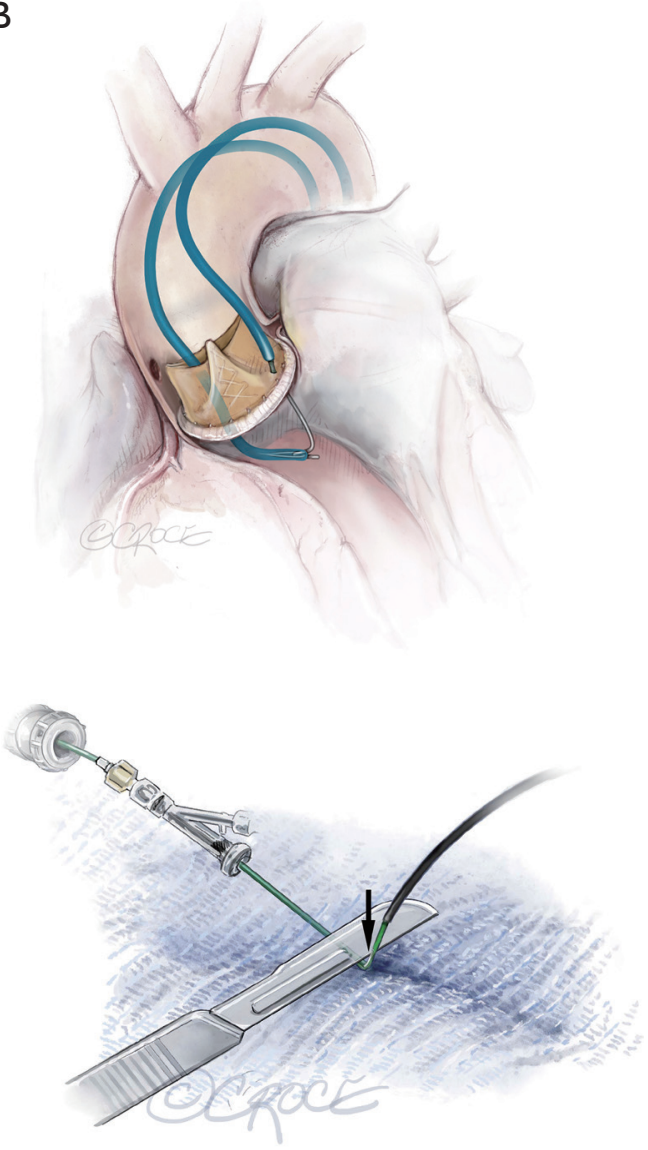

Figure 1 Electrosurgical leaflet traversal and snaring. Access is with a 14-Fr sheath (DrySeal Flex, Gore, Flagstaff, AZ) in the artery selected for TAVR and a 6-Fr sheath in the contralateral femoral artery, traditionally utilized for pigtail angiography. One BASILICA guiding catheter is inserted through each sheath. (A) The traversal catheter (designated as the catheter through which the leaflet traversal wire will be delivered) is inserted through the $14 \mathrm{Fr}$ Gore sheath, advanced to and positioned on the target aortic leaflet. We recommend an oversized 7 Fr Amplatz left (e.g., AL2, AL3) guiding catheter for the left and 7 Fr Judkins right (JR4) for the right. In a minority of cases a coaxial $125 \mathrm{~cm} 5 \mathrm{Fr}$ internal mammary (IM1) or JR4 shape diagnostic catheter through the AL2/3 in mother-daughter configuration may be required for left leaflet traversal. Optimal positioning is achieved when the guiding catheter rests on the center of the leaflet base, points towards the left ventricular outflow tract (LVOT) and often 'bounces' on the leaflet. Positioning is confirmed with orthogonal fluoroscopic projections (side and en face) and with a small volume contrast injection. The second guiding catheter (6 Fr JR4/Multipurpose) is positioned in the LVOT, stabilized with a 0.018 " guidewire (e.g., Steelcore, Abbott Vascular, North Plymouth, $\mathrm{MN}$ ) and directed to present a single loop snare (20-30 mm Amplatz Gooseneck) just below and parallel to the aortic annulus. A 300-cm angioplasty guidewire (Astato XS20, Asahi Intecc USA, Santa Ana, CA) is inserted in a 145-cm hubless locking 0.014" microcatheter (Piggyback wire converter, Teleflex Vascular Solutions, Minneapolis, $\mathrm{MN}$ ) and advanced within the crossing guide catheter until it reaches the leaflet. The proximal end of the guidewire is manually denuded and connected to an electrosurgery pencil (and generator set at 30-50 W "pure" cut) by cross-clamping with hemostatic forceps. Tactile resistance is encountered and the leaflet may be 'palpated' to ensure good apposition. It is imperative to ensure that only the very distal tip of the guidewire extends beyond the microcatheter. Positioning is again confirmed fluoroscopically. The traversal wire is energized briefly $(<1$ sec) whilst being advanced towards the pre-positioned LVOT snare. (B) The guidewire is snared by and invaginated into the LVOT catheter where it is retained. (C) The microcatheter is removed from the body, positioned approximately one hand's breadth from the proximal end of the guidewire and locked. The 'flying $\mathrm{V}$ ' is thereafter fashioned by non-circumferentially denuding a short segment of the guidewire's mid shaft with a scalpel blade at the distal tip of the microcatheter (upper panel). The scalpel is inverted and the blunt edge used to kink the wire to $180^{\circ}$. A residual kink angle of approximately $150^{\circ}$ is retained (lower panel). 
A

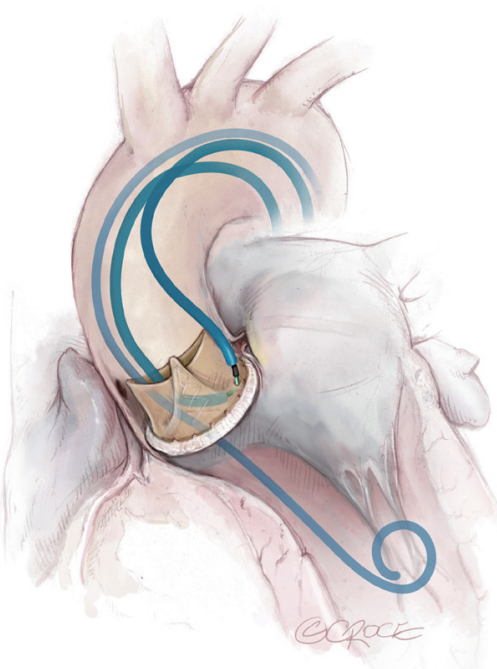

C

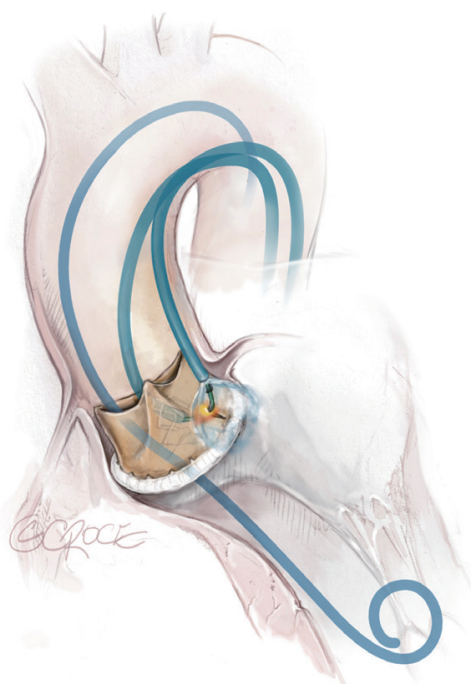

B

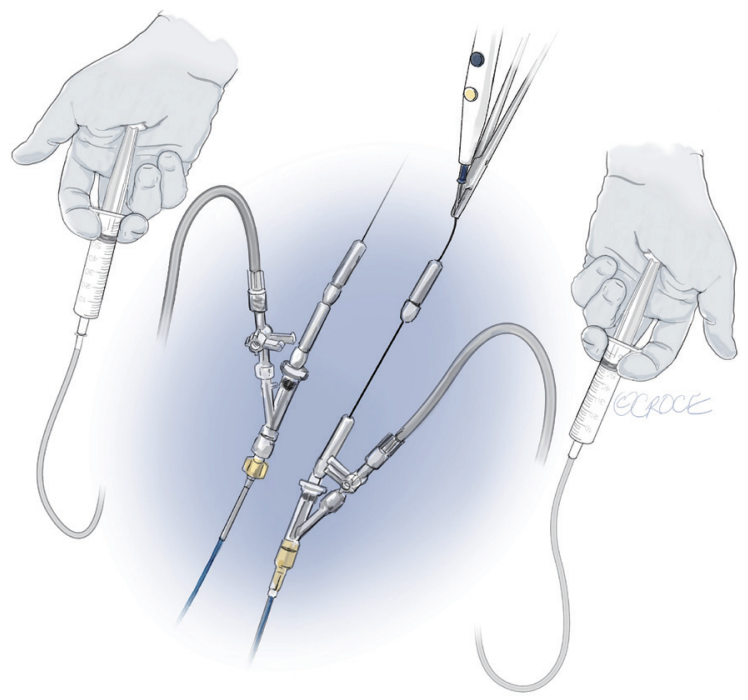

D

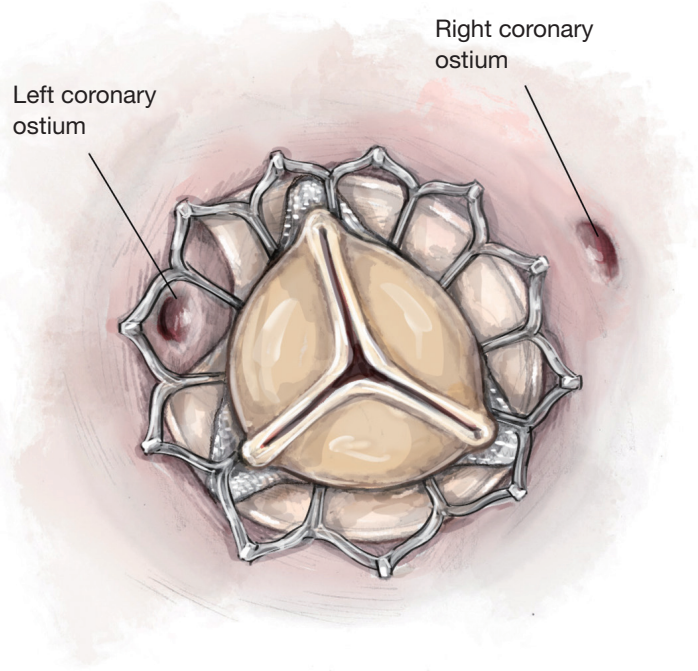

Figure 2 Leaflet laceration. (A) The 'flying $\mathrm{V}$ ' is advanced within the traversal catheter while externalizing the snared distal wire through the snare catheter. It may be necessary to temporarily detach the rotating hemostatic valve from the traversal catheter to deliver the kink in to the catheter hub. It is important to insert the snare loader to the hemostatic valve on the snare catheter to prevent inadvertent loss of the snared wire within the valve. The flying $\mathrm{V}$ is positioned fluoroscopically at the base of the leaflet. Care is taken to ensure both guiding catheters are advanced to the leaflet base, one on the aortic side, the other on the LVOT surface. A pigtail catheter is inserted through the DrySeal sheath and pre-positioned in the left ventricle in preparation for TAVR. (B) In preparation for tensioning of the system, a series of wire torquers are attached to the external wire and microcatheter shafts. An 0.035 " rotating torquer is inserted over the locking microcatheter to abut the rotating hemostatic valve and tightened. Two 0.014 " torquers are passed over the snared distal wire limb and one over the proximal wire (three in total) to abut the junction with the microcatheter and tightened. Two 50 cc syringes filled with $5 \%$ dextrose are attached to the side arms of the rotating hemostatic valves and the catheters are flushed. This key step replaces the ionic blood-filled column with non-ionic dextrose and permits charge concentration on the leaflet during electrosurgical laceration when flushed. (C) Slack is removed from the system by applying gentle traction on the coaxial guiding catheter systems. The catheters are visualized hugging the inner curvature of the aorta and the operator feels resistance when adequately positioned. Tension is maintained on both guiding catheters. The guidewire is energized at $70 \mathrm{~W}$ pure cut whilst flushing dextrose generously through both guiding catheters. When leaflet laceration is complete, guiding catheters will be observed 'jumping' towards the aortic arch and energy delivery is stopped immediately. (D) Completed laceration showing midline slit and unobstructed left coronary ostium. 
A

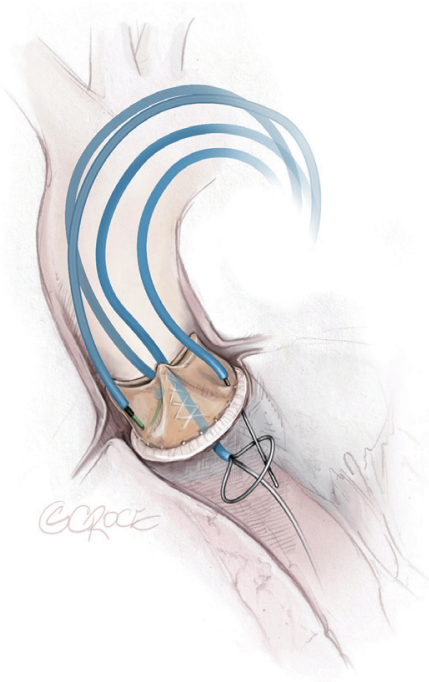

B

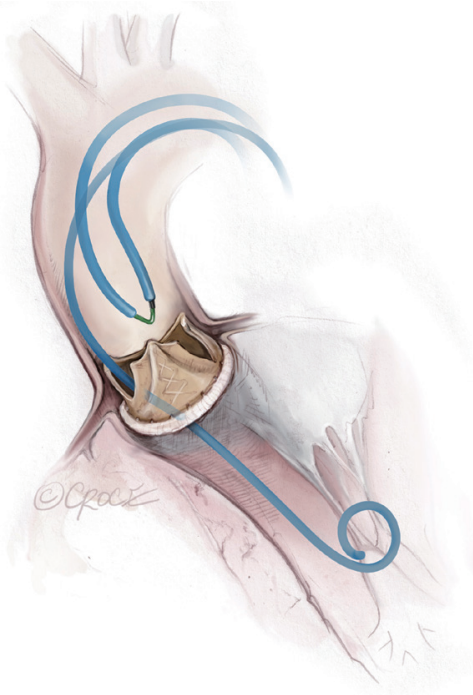

C

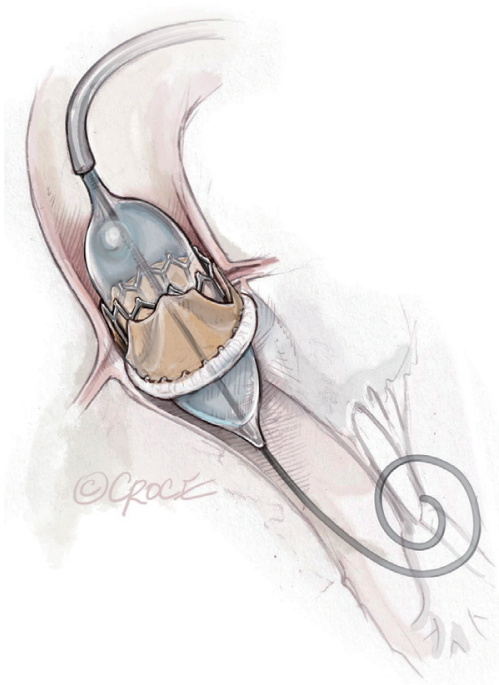

Figure 3 Double leaflet (Doppio) BASILICA. Access is with two 14-Fr femoral arterial sheaths (e.g., DrySeal Flex) that permit introduction of multiple parallel catheters whilst maintaining hemostasis. (A) One pair of BASILICA catheters (traversal and snare) are introduced through each access sheath. Leaflets are crossed and snared sequentially at the operator's discretion. Flying V kinks are formed on both wires as described above (Figure 1) and are delivered in turn to their target leaflets. Coaxial systems are aligned and torquers and dextrose syringes attached to the guiding catheters and hemostatic valves. (B) One leaflet is lacerated with application of $70 \mathrm{~W}$ 'pure' cut energy and continuous dextrose flush. Prior to laceration of the second leaflet a pigtail is placed in the left ventricle in preparation for TAVR and the 14-Fr sheath is exchanged for the TAVR delivery sheath. The second leaflet is lacerated and BASILICA catheters are removed from the body. (C) TAVR is performed in traditional fashion.

concentrate electrosurgical energy during leaflet traversal or laceration.

\section{Failure to traverse or lacerate}

\section{(I) Ineffective delivery of radiofrequency energy}

Connections between electrosurgical generator and pencil should be checked to ensure it is inserted correctly and that it is connected to the cut function (yellow), not coagulation function (blue). The proximal end of the guidewire should be denuded effectively to remove all insulative polytetrafluoroethylene (PTFE) (green) coating. Cross clamping of the guidewire to the electrosurgical pencil should be secure-the wire should not move from the jaws of the hemostat when tugged. The second operator should be aware to press the yellow "cut" button during application of energy, not the blue "coagulation" button. Energy should be appropriately selected-generally 30-50 W for leaflet traversal and $70 \mathrm{~W}$ for leaflet laceration.

\section{Failure to traverse}

(I) Position of traversal catheter

Catheter positioning on the surgical aortic valve sewing ring may be misinterpreted as appropriate placement. Subsequent attempts at wire traversal will fail as it advances in to the ring rather than across leaflet tissue. Visual cues that may differentiate ring from leaflet engagement include an undulation of the catheter tip (moving synchronously with the annulus) suggestive of sewing ring engagement as opposed to bouncing (paradoxical movement of the catheter tip and target leaflet). If encountered, the traversal guide catheter should be repositioned until it bounces or it may require to be 'upsized' (e.g., from AL2 to AL3).

\section{(II) Positioning of coaxial crossing system}

Care should be taken to ensure that the coaxial system of guiding catheter, microcatheter and guidewire is positioned orthogonal to the base of the target leaflet prior to attempted traversal. If this is not achieved the wire may be visualized 'skidding' tangentially over the leaflet surface towards the annulus.

\section{(III) Calcium at traversal target}

Dense calcification of the leaflet base at the intended crossing point may prevent energy transfer and vaporization 
A

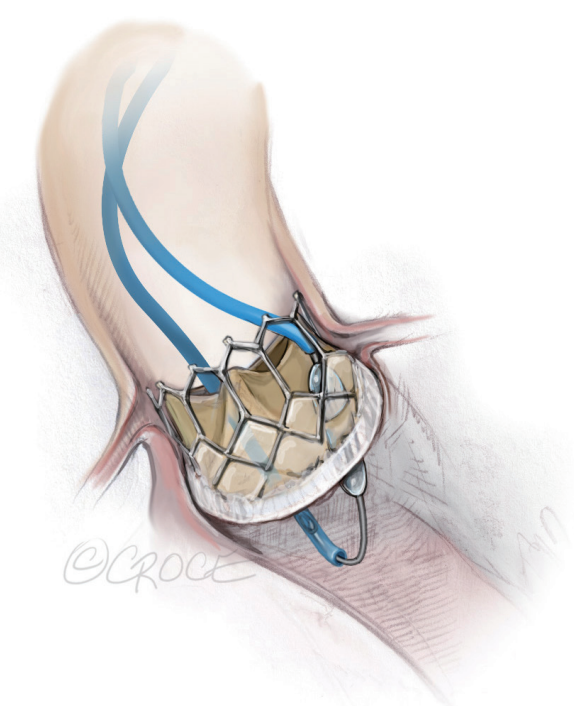

B

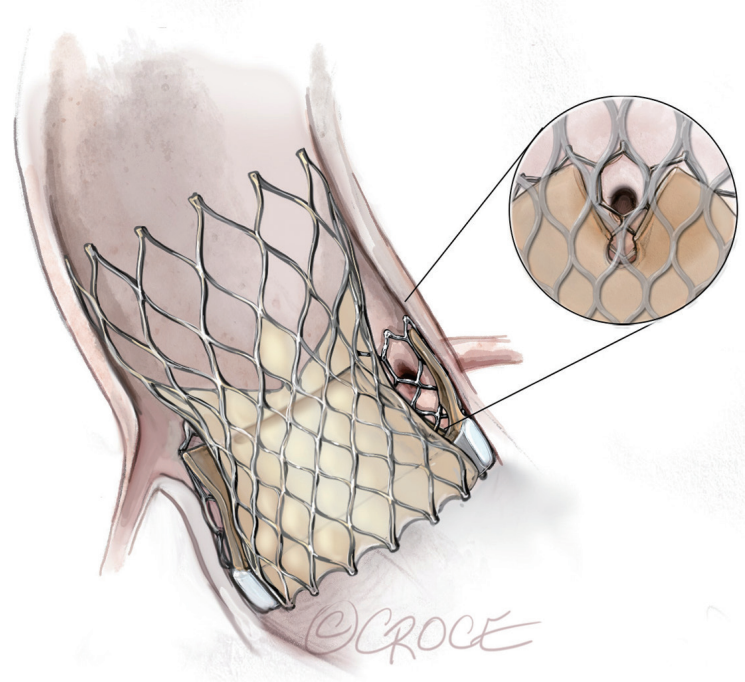

Figure 4 Balloon-augmented (BA) BASILICA. Access is identical to traditional BASILICA depending on whether a single or Doppio procedure is to be performed. (A) The coaxial system comprising 7-Fr traversal guiding catheter, Piggyback wire converter and Astato XS20 guidewire is advanced to and positioned on the base of the target leaflet. A single loop snare, sized to the LVOT, is positioned below the aortic annulus through a 6-Fr guiding catheter (JR4/Multipurpose). Leaflet traversal is performed during brief ( $<1 \mathrm{~s})$ application of 30-50 W "pure" cut RF energy and the wire is snared and retained in the LVOT. The Piggyback is removed and replaced with a 4.5-5.0 mm noncompliant, rapid exchange ( $\mathrm{Rx}$ ) angioplasty balloon. The balloon is positioned to straddle the base of the target leaflet and inflated to dilatate the leaflet crossing point. The balloon is subsequently removed, locking microcatheter re-inserted over the proximal shaft of the wire and flying $\mathrm{V}$ created. The flying $\mathrm{V}$ is positioned at the point of dilatation in the leaflet base and laceration performed. (B) A large leaflet splay is created, further reducing the likelihood of coronary artery obstruction in high risk cases e.g., TAV-in-TAV.

of tissue. The wire will deflect and should be repositioned. Cases with dense, confluent calcification at the leaflet base may not be appropriate for BASILICA procedures.

\section{Failure to lacerate}

When charge concentration is adequate, steady maintenance of gentle traction will result in leaflet laceration. If charge fails to concentrate the operator should avoid increasing force using a 'lawnmower pull cord' maneuver and instead halt and evaluate the system concentrating on the following areas.

\section{(I) Ineffective dextrose flush}

The displacement of ionic blood by non-ionic 5\% dextrose permits effective charge concentration at the lacerating surface by removing alternative current paths (10). It is also beneficial in preventing accumulation of char and thrombus at the cutting surface. The operator should ensure all 3-way connectors are open to the catheter and that rate of flush is satisfactory.

\section{(II) Microcatheter displacement during traction}

When applying traction to the catheter systems the operator should be vigilant to the positioning of the insulating microcatheters, displacement of which will allow for current dispersal (10). If microcatheter slippage is observed, energy delivery should be stopped immediately. The microcatheter locking mechanism should be inspected to ensure it is closed in the lock position. All torquers should be adjusted to a position of close apposition with microcatheter, wire or rotating hemostatic valve as appropriate and re-tightened.

\section{Comments}

\section{Clinical results}

The BASILICA IDE trial reported thirty-day and oneyear results in a cohort of thirty prohibitive risk patients with multiple comorbidities undergoing BASILICA $(11,12)$. Procedural success was reported in twenty-eight patients, 
disabling stroke in one and no early or late coronary obstruction. Real world data from the international BASILICA registry including 214 patients from twentyfive centers demonstrated that BASILICA was feasible with successful leaflet traversal and laceration in $94.4 \%$. Doppio procedures were performed in $21.5 \%$. At thirty days disabling stroke was reported in only $0.5 \%, 95.3 \%$ had freedom from culprit coronary artery obstruction and survival was $97.2 \%$.

\section{Advantages}

Alternative approaches to prevent or treat coronary artery obstruction are surgery and snorkel/"chimney" stenting. Surgery is a good option in patients of low or intermediate surgical risk and should remain a consideration during Heart Team discussions. Higher surgical risk patients currently have only two options-BASILICA or snorkel stenting. Snorkel stents are deployed between the TAVR frame and the aortic sinus or root. These stents are often under-expanded and risk being completely crushed which carries a high risk of acute or delayed coronary artery obstruction (13-15). Patients may remain on long-term dual antiplatelet therapy or anticoagulant which is undesirable in a population that tends to be elderly with multiple co-morbidities. Future coronary artery access will be challenging or impossible.

BASILICA can be performed with readily available, 'off the shelf' equipment, which negates the requirement for prolonged dual antiplatelet therapy, has an enlarging body of real world feasibility and safety data, and maintains coronary artery access in an orthotopic fashion for future de novo disease. Although the skill set may be unfamiliar to many operators, independent practice is expected following two to three proctored single leaflet cases.

\section{Caveats}

Several clinical situations require caution when considering BASILICA as they may risk coronary ostial obstruction despite successful leaflet laceration. Leaflet splay may be reduced during TAV-in-TAV procedures as the lacerated leaflet becomes pinned by the outer transcatheter heart valve frame (2). Moreover, BASILICA will not prevent coronary obstruction when stent posts of the degenerative transcatheter heart valve (THV) lie in front of a coronary ostium and are pushed outwards during delivery of the second valve (5). Eccentric coronary ostia may obstruct despite successful midline BASILICA laceration. Uncertainty remains as to the degree of leaflet splay experienced in vivo in patients with severely calcified and degenerative valve leaflets.

\section{Future directions}

Purpose-built guidewires, catheters and adjunctive tools are required to further simplify BASILICA techniques. Pachyderm-shaped guiding catheters (PAL1-3, PJR4) have already reduced time-to-leaflet traversal and with fewer crossing attempts per leaflet when compared to traditional techniques (16).

Mechanical leaflet laceration has recently been described with the ShortCut device (Pi-Cardia, Israel) but ability to cut through calcified leaflets is currently unknown. Excision of degenerative native or bioprosthetic valve leaflets is also an attractive concept, particularly in patients with eccentric coronary ostia, bulky leaflet calcification or who risk coronary obstruction following TAV-in-TAV with sinus effacement.

\section{Acknowledgments}

Funding: Supported by the Division of Intramural Research, National Heart, Lung and Blood Institute, National Institutes of Health, USA (Z01-HL006040).

\section{Footnote}

Conflicts of Interest: ABG-Proctor for Edwards Lifesciences, Medtronic, and Abbott Vascular and he is a consultant for Transmural Systems. VCB-Consultant for Edwards Lifesciences and for Abbott Vascular, and his employer has research contracts for clinical investigation of transcatheter aortic and mitral devices from Edwards Lifesciences, Abbott Vascular, Medtronic, St Jude Medical, and Boston Scientific. TR-Consultant and proctor for Edwards Lifesciences, Medtronic. On the Advisory Board for Medtronic. He has Equity Interest in Transmural Systems. JMK, TR, and RJL are co-inventors on patents, assigned to NIH, on catheter devices to lacerate valve leaflets. NHLBI has a collaborative research and development agreement with Edwards Lifesciences on transcatheter modification of the mitral valve. CGB has no conflicts of interest to declare.

Open Access Statement: This is an Open Access article distributed in accordance with the Creative Commons 
Attribution-NonCommercial-NoDerivs 4.0 International License (CC BY-NC-ND 4.0), which permits the noncommercial replication and distribution of the article with the strict proviso that no changes or edits are made and the original work is properly cited (including links to both the formal publication through the relevant DOI and the license). See: https://creativecommons.org/licenses/by-nc-nd/4.0/.

\section{References}

1. Ribeiro HB, Webb JG, Makkar RR, et al. Predictive factors, management, and clinical outcomes of coronary obstruction following transcatheter aortic valve implantation: insights from a large multicenter registry. $\mathrm{J}$ Am Coll Cardiol 2013;62:1552-62.

2. Khan JM, Bruce CG, Babaliaros VC, et al. TAVR Roulette: Caution Regarding BASILICA Laceration for TAVR-in-TAVR. JACC Cardiovasc Interv 2020;13:787-9.

3. Ribeiro HB, Rodés-Cabau J, Blanke P, et al. Incidence, predictors, and clinical outcomes of coronary obstruction following transcatheter aortic valve replacement for degenerative bioprosthetic surgical valves: insights from the VIVID registry. Eur Heart J 2018;39:687-95.

4. Forrestal BJ, Case BC, Yerasi C, et al. Risk of Coronary Obstruction and Feasibility of Coronary Access After Repeat Transcatheter Aortic Valve Replacement With the Self-Expanding Evolut Valve: A Computed Tomography Simulation Study. Circ Cardiovasc Interv 2020;13:e009496.

5. Rogers T, Greenspun BC, Weissman G, et al. Feasibility of Coronary Access and Aortic Valve Reintervention in Low-Risk TAVR Patients. JACC Cardiovasc Interv 2020;13:726-35.

6. Khan JM, Dvir D, Greenbaum AB, et al. Transcatheter Laceration of Aortic Leaflets to Prevent Coronary Obstruction During Transcatheter Aortic Valve Replacement: Concept to First-in-Human. JACC Cardiovasc Interv 2018;11:677-89.

Cite this article as: Bruce CG, Greenbaum AB, Babaliaros VC, Rogers T, Lederman RJ, Khan JM. Safeguards and pitfalls for Bioprosthetic or Native Aortic Scallop Intentional Laceration to Prevent Iatrogenic Coronary Artery Obstruction during transcatheter aortic valve replacement-the BASILICA technique. Ann Cardiothorac Surg 2021;10(5):700-707. doi: 10.21037/acs-2021-tviv-26
7. Lederman RJ, Babaliaros VC, Rogers T, et al. Preventing Coronary Obstruction During Transcatheter Aortic Valve Replacement: From Computed Tomography to BASILICA. JACC Cardiovasc Interv 2019;12:1197-216.

8. Khan JM, Babaliaros VC, Greenbaum AB, et al. Preventing Coronary Obstruction During Transcatheter Aortic Valve Replacement: Results From the Multicenter International BASILICA Registry. JACC Cardiovasc Interv 2021;14:941-8.

9. Greenbaum AB, Kamioka N, Vavalle JP, et al. BalloonAssisted BASILICA to Facilitate Redo TAVR. JACC Cardiovasc Interv 2021;14:578-80.

10. Khan JM, Rogers T, Greenbaum AB, et al. Transcatheter Electrosurgery: JACC State-of-the-Art Review. J Am Coll Cardiol 2020;75:1455-70.

11. Khan JM, Greenbaum AB, Babaliaros VC, et al. The BASILICA Trial: Prospective Multicenter Investigation of Intentional Leaflet Laceration to Prevent TAVR Coronary Obstruction. JACC Cardiovasc Interv 2019;12:1240-52.

12. Khan JM, Greenbaum AB, Babaliaros VC, et al. BASILICA Trial: One-Year Outcomes of Transcatheter Electrosurgical Leaflet Laceration to Prevent TAVR Coronary Obstruction. Circ Cardiovasc Interv 2021;14:e010238.

13. Jabbour RJ, Tanaka A, Finkelstein A, et al. Delayed Coronary Obstruction After Transcatheter Aortic Valve Replacement. J Am Coll Cardiol 2018;71:1513-24.

14. Mercanti F, Rosseel L, Neylon A, et al. Chimney Stenting for Coronary Occlusion During TAVR: Insights From the Chimney Registry. JACC Cardiovasc Interv 2020;13:751-61.

15. Pighi $M$, Lunardi $M$, Pesarini $G$, et al. Intravascular ultrasound assessment of coronary ostia following valve-in-valve transcatheter aortic valve implantation. EuroIntervention 2021;16:1148-51.

16. Lisko JC, Babaliaros VC, Lederman RJ, et al. PachydermShape Guiding Catheters to Simplify BASILICA Leaflet Traversal. Cardiovasc Revasc Med 2019;20:782-5. 